\title{
Simultaneous measurement of resistance and temperature changes in bridge circuits
}

\author{
Klemens Gintner \\ University of Applied Sciences Karlsruhe, Faculty MMT, Moltkestrasse 30, 76133 Karlsruhe, Germany
}

\begin{abstract}
Wheatstone Bridge Circuits are commonly used in sensor applications to measure the resistance change of sensor elements, which represent typical metrics such as pressure, force or magnetic flux density. In many applications, for example, magnetoresistive or pressure-sensitive sensor elements are used in Wheatstone bridges. A temperature change of the Wheatstone bridge leads not only to a change of the resistance of the sensor elements, but also of the reference resistors. The reason for this is the (mostly positive) temperature coefficient of the resistors and also the dependence of the sensor effect on the temperature. In order to obtain a more accurate result, the temperature of the bridge circuit should therefore be known. By using several different voltages in a bridge circuit and knowing the temperature coefficients, it is possible to determine the exact resistance change of the sensor elements and the temperature change simultaneously without using an additional temperature sensor. A possible offset of the bridge circuit due to a mismatch of the four resistors is not relevant.
\end{abstract}

Keywords: Bridge-Circuit, Temperature, resistance change

\section{Method for determining the resistances in a Wheatstone bridge under reference conditions}

In this proposal it is essential to know the exact values of the four resistances in the Wheatstone bridge under reference conditions, i.e. the temperature is given; then the temperature change is zero $(\mathrm{dT}=0$, therefore the temperature coefficients $a$ and $b$ are not relevant) and there is no change in resistance as no measured quantity is applied (then: $d R=0$ ).

To accurately measure each of the four resistors, a floating zero-resistance ammeter is suggested, as shown in Figure $1 \mathrm{a}$ and $1 \mathrm{~b}$. It is important to note that the bridge circuit with R1 to R4 has already been produced and is ready for use. The basic idea is to short-circuit one of the two resistors connected in series connection (in Figure 1a and 1b: node 1 and node 2 are short-circuited so that R4 is no longer relevant) and measure the current (here: I(R3)) using operational amplifiers so that, for example, the resistance of R3 can be determined by the voltage $U_{3}$ (then $U_{3}=U v$ ) divided by the measured current I( $\left.\mathrm{R}_{8}\right)$; here: $I\left(R_{8}\right)=I\left(R_{3}\right)$ with $I\left(R_{3}\right)$ as the current through the (reference) resistor R3 in the Wheatstone bridge; all this is resulting in: $R 3=U_{3} / I\left(R_{3}\right)=U_{V} / I\left(R_{8}\right)$. The current $I\left(R_{8}\right)$ $\left(=I\left(R_{7}\right)\right)$ can be derived by using the difference of the voltages at the output of the two operational amplifiers $\left(U_{A 1}-U_{A 2}\right)$ according to equation (1); it is important to know that $\left(R_{5} \cdot R_{7}\right)$ is equal to $\left(R_{6} \cdot R_{8}\right)$.

Equation (1) for $I\left(R_{3}\right)$ results in:

$$
I\left(R_{3}\right)=\frac{U_{A 1}-U_{A 2}}{R_{7} \cdot\left(1+R_{5} / R_{6}\right)}=I\left(R_{8}\right)=I\left(R_{7}\right)
$$

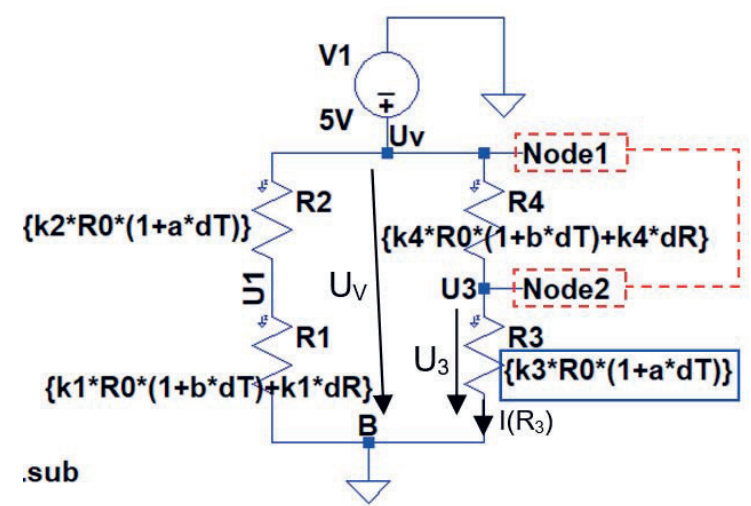

Fig. 1a: Floating zero-resistance ammeter for measuring the resistances at reference conditions $(\mathrm{dR}=0$ and $\mathrm{dT}=0$ ) (for further details see [1])

In this example $k 3^{*} R_{0}$ is the value of the resistance at $\mathrm{dT}=0: \mathrm{R} 3=\mathrm{U}_{\mathrm{v}} / \mathrm{I}(\mathrm{R} 3)=1.05 \mathrm{k} \Omega$ which corresponds with the correction factor k3 $=1.05$ multiplied with the nominal resistance $\mathrm{R} 0=1 \mathrm{k} \Omega$. All this can be realised at the end of the production process and possibly the data can be stored in the sensor (e.g. by means of laser fuses) and can be used in signal conditioning circuits. With a micro- 
controller the change of the resistance $\mathrm{dR}$ and the change of the temperature dT can be evaluated as described in the following chapters.

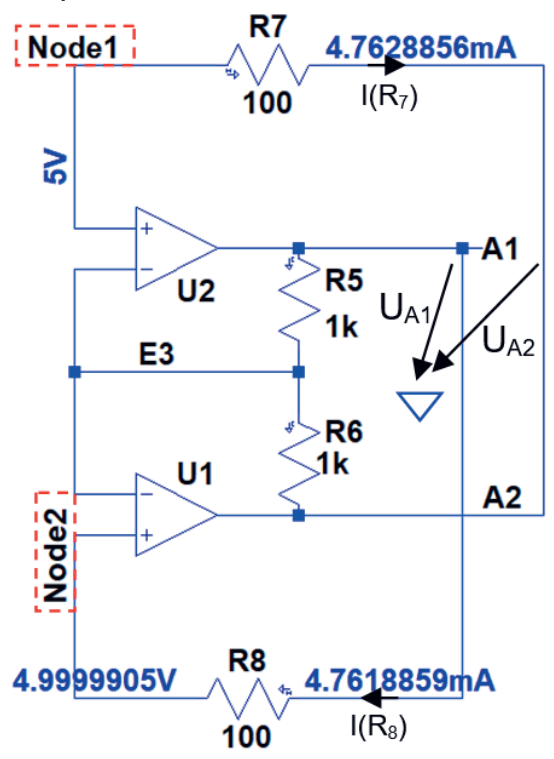

Fig. 1b: Floating zero-resistance ammeter for measuring the resistances at reference conditions $(d R=0$ and $d T=0$ ) (for further details see [1])

\section{Method for simultaneously determining the change in resistances and temperature in Wheatstone bridges supplied with a voltage source}

A new concept for the simultaneous determination of the change in resistance $\mathrm{dR}$ of sensor elements and the change in temperature $\mathrm{dT}$ in Wheatstone bridges is proposed. The basic idea is to consider the known temperature coefficients of all resistors used in the bridge circuit. Then the absolute change of the resistance $\mathrm{dR}$ and the temperature $\mathrm{dT}$ compared to the reference conditions (with $\mathrm{dR}=0$ and $\mathrm{dT}=0$ ) can be determined by using different voltages of the bridge circuit. The equations depend on the circuit, so a quarter bridge with one sensor element, a half bridge with two sensor elements or a full bridge circuit with four sensor elements have different equations. For each sensor element, the relative change of the resistance $\mathrm{dR} / \mathrm{R}_{0}$ is the same when the same measured variable is applied, where $R_{0}$ represents the nominal resistance at reference conditions.

\subsection{Quarter bridge circuit with one sensor element and voltage source}

In the quarter bridge depicted in Figure 2, the sensor element is represented by the resistor $\mathrm{R} 1 ; \mathrm{R}_{0}$ is the nominal value (here: $1 \mathrm{k} \Omega$ ) and $\mathrm{k} 1$ is the factor that determines the exact value of $\mathrm{R} 1$ at reference conditions same is valid for k2 for R2, k3 for R3 and k4 for $\mathrm{R} 4$; $\mathrm{b}$ is the constant temperature coefficient of $\mathrm{R} 1$ and $\mathrm{dR}$ represents the absolute change of $\mathrm{R} 1$ due to the measured variable. The resistors R2, R3 and R4 are reference resistors with different constant temperature coefficients (TC) $a$ and $g$ with $a \neq g$. It is possible: $a=b$ or $g=b$ with $b$ as TC of the sensor element; this means that either R3 or (R2 and R4) can be replaced by an insensitive sensor element which only reacts to temperature changes but not to changes in the measured variable. But always the temperature coefficients a and $g$ of R3 and R4 need to be different: $\mathrm{a} \neq \mathrm{g}$.

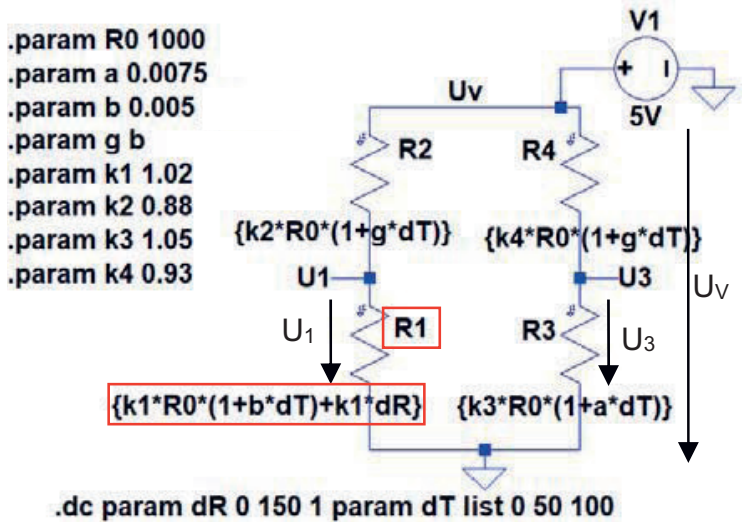

Fig. 2: Quarter bridge circuit with one sensor element (R1) and three reference resistors (R2, $R 3, R 4)$

Sensor resistance:

$R_{1}=k_{1} \cdot R_{0} \cdot(1+b \cdot d T)+k_{1} \cdot d R$

Reference resistances:

$R_{2}=k_{2} \cdot R_{0} \cdot(1+g \cdot d T)$

$R_{3}=k_{3} \cdot R_{0} \cdot(1+a \cdot d T)$

$R_{4}=k_{4} \cdot R_{0} \cdot(1+g \cdot d T)$

In equation (2) the calculation of the temperature change dT is shown; equation (3) describes how the relative change of the resistance $\mathrm{dR} / \mathrm{R}_{0}$ of the sensor element can be derived with $\mathrm{R}_{0}$ as nominal resistance.

Figure 3 shows the result of this calculation in a simulation with LTspice [3]. It can be seen that both the temperature change $\mathrm{dT}$ and the relative resistance change $d R / R_{0}$ can be derived simultaneously using equations (2) and (3).

For the change of the temperature dT equation (2) is used:

$$
d T=\frac{k_{3} \cdot U_{V}-U_{3} \cdot\left(k_{3}+k_{4}\right)}{U_{3} \cdot\left(a \cdot k_{3}+g \cdot k_{4}\right)-a \cdot k_{3} \cdot U_{V}}
$$

and equation (3) for the relative change $d R / R_{0}$ of the resistance of the sensor element $\mathrm{R} 1$ : 


$$
\begin{aligned}
\frac{d R}{R_{0}}= & \frac{\left[k_{3} \cdot U_{V}-U_{3} \cdot\left(k_{3}+k_{4}\right)\right]}{\left(U_{V}-U_{1}\right) \cdot k_{1}} . \\
& \cdot \frac{\left[k_{2} \cdot g \cdot U_{1}-k_{1} \cdot b \cdot\left(U_{V}-U_{1}\right)\right]}{\left[U_{3} \cdot\left(a \cdot k_{3}+g \cdot k_{4}\right)-a \cdot k_{3} \cdot U_{V}\right]} \\
- & \frac{\left(k_{1} \cdot\left(U_{V}-U_{1}\right)-k_{2} \cdot U_{1}\right)}{\left(U_{V}-U_{1}\right) \cdot k_{1}} \cdot \\
& \cdot \frac{\left[U_{3} \cdot\left(a \cdot k_{3}+g \cdot k_{4}\right)-a \cdot k_{3} \cdot U_{V}\right]}{\left[U_{3} \cdot\left(a \cdot k_{3}+g \cdot k_{4}\right)-a \cdot k_{3} \cdot U_{V}\right]}
\end{aligned}
$$

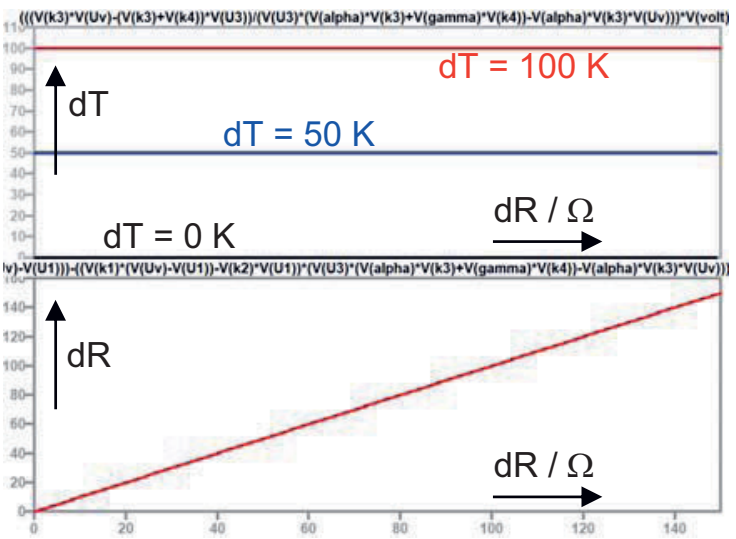

Fig. 3: Quarter bridge circuit (see Fig. 2) depending on change of $R(d R)$ with temperature change $d T$ as parameter: Top: Temperature change $d T$; Bottom: Resistance change $d R$

\subsection{Half bridge circuit with two sensor elements and voltage source}

In the half bridge shown in Figure 4 two sensor elements R1 and R4 are used. The resistors $\mathrm{R} 2$ and $\mathrm{R} 3$ represent the reference resistors with different temperature coefficients (TC) a and $\mathrm{g}: \mathrm{a} \neq \mathrm{g}$.

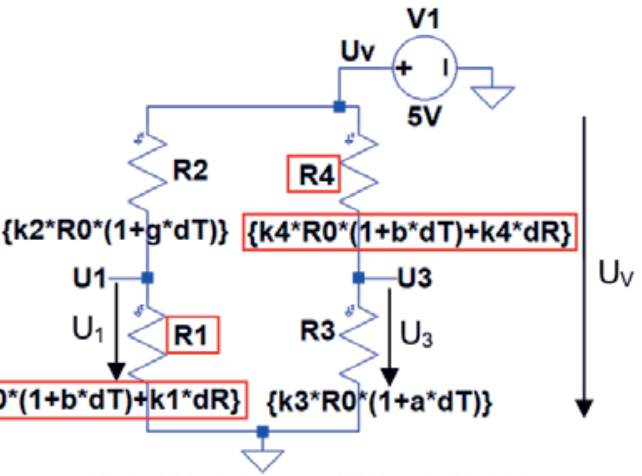

.dc param dR 01501 param dT list 050100

Fig. 4: Half bridge circuit with two sensor elements $(R 1, R 4)$ and two reference resistors $(R 2, R 3)$
Similar to the previous chapter, $d R$ (or $d R / R_{0}$ ) and $\mathrm{dT}$ can be calculated using equations (4) and (5). In this example the following also applies: $g=b$; so $\mathrm{R} 2$ can be realised as an insensitive sensor element. It is important that the TC of the reference resistors $\mathrm{R} 2$ and $\mathrm{R} 3$ are different: $a \neq g$.

Equation (4):

$$
\begin{aligned}
d T= & \frac{k_{4} \cdot U_{3} \cdot\left[k_{2} \cdot U_{1}-k_{1} \cdot\left(U_{V}-U_{1}\right)\right]+}{k_{1} \cdot\left(U_{V}-U_{1}\right) \cdot\left[k_{3} \cdot a \cdot\left(U_{V}-U_{3}\right)-k_{4} \cdot b \cdot U_{3}\right]+} \\
& \ldots \frac{-k_{1} \cdot\left(U_{V}-U_{1}\right) \cdot\left[k_{3} \cdot\left(U_{V}-U_{3}\right)-k_{4} \cdot U_{3}\right]}{+k_{4} \cdot U_{3} \cdot\left[k_{1} \cdot b \cdot\left(U_{V}-U_{1}\right)-k_{2} \cdot g \cdot U_{1}\right]}
\end{aligned}
$$

Equation (5):

$$
\begin{gathered}
\frac{d R}{R_{0}}=\frac{k_{3} \cdot\left(U_{V}-U_{3}\right) \cdot(1+a \cdot d T)}{k_{4} \cdot U_{3}}- \\
\frac{k_{4} \cdot U_{3} \cdot(1+b \cdot d T)}{k_{4} \cdot U_{3}}
\end{gathered}
$$

The results of equations (4) and (5) are depicted in Figure 5; again, the change in temperature dT and the change in resistance $\mathrm{dR}$ of the sensor element can be calculated using equation (4); then the change in resistance $\mathrm{dR}$ can be derived using equation (5).

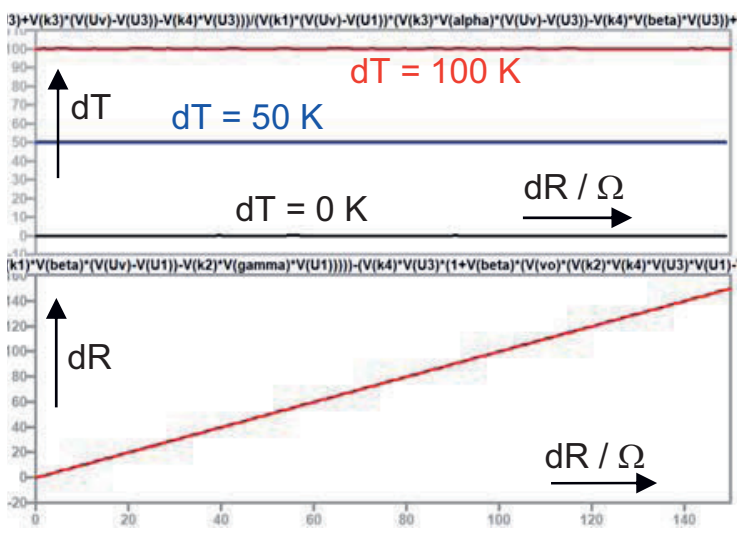

Fig. 5: Half bridge circuit (see Fig. 4) depending on change of $R(d R)$ with temperature change $d T$ as parameter: Top: Temperature change dT Bottom: Resistance change $d R$

\section{Method for simultaneously determining the resistance and temperature change of Wheatstone bridges with additional resistor and voltage source}

If there is an additional resistor $\mathrm{R} 5$ used that is connected in series to the bridge circuit (see Figure 6), it is also feasible to calculate the change of the temperature dT and the change of the resistance $\mathrm{dR}$ of the sensor elements. The idea is to get the information about the 
total current flowing through the bridge circuit in order to calculate $\mathrm{dT}$ and $\mathrm{dR}$. This is shown in the following chapters for quarter bridge, half bridge and full bridge Wheatstone circuit.

\subsection{Quarter bridge circuit with one sensor element and additional series resistor}

Figure 6 shows a quarter bridge circuit (with one sensor element R1 and three reference resistors R2, R3, R4) with an additional resistor R5. As in the previous chapters, it is possible to derive the temperature change dT and the resistance change $\mathrm{dR}$ (or relative change $d R / R_{0}$ ) of the sensor element by using voltages, temperature coefficients (TC) and resistances at reference conditions - see equations (6) und (7); the temperature change dT as a result of equation (6) is used in equation (7). Here the temperature coefficients a of the three reference resistors may be similar (without R5 they need to be different, see chapter 2.1) - the temperature coefficient a can be similar to the temperature coefficient $b$ of the sensor element R1; therefore: $\mathrm{a}=\mathrm{b}$. If $\mathrm{R} 5$ is not constant, then the temperature coefficient $f$ (with $R 5=$ $k 5^{*} R 0^{*}\left(1+f^{*} d T\right)$, see Fig. $\left.6: f \neq a\right)$ and the temperature of R5 must be known; it is still possible: $a=b$. R5 may be located in close proximity to the signal conditioning circuit (e.g. microcontroller or electronic control unit); the temperature change and hence the change of $\mathrm{R} 5$ is then usually limited.

$d T=\frac{\frac{k_{3}}{k_{2}} \cdot U_{2}+U_{3}}{a \cdot\left(U_{V}-U_{B R}\right) \cdot \frac{k_{3} \cdot R_{0}}{R_{5}}}-\frac{1}{a}(6)$, with R5 as constant resistor and equation (7):

$\frac{d R}{R_{0}}=\frac{k_{2}}{k_{1}} \cdot(1+a \cdot d T) \cdot\left(\frac{U_{1}}{U_{B R}-U_{1}}\right)-(1+b \cdot d T)$

In Figure 7 the result of the simulation with LTspice [3] is shown - again the change of the temperature dT and the change of the resistance $\mathrm{dR}$ of the sensor element can be calculated by using different voltages and temperature coefficients.

If the voltage source $\mathrm{V} 1$ and the resistor R5 are substituted by a current source $\mathrm{I}_{5}$, equation (6) for changing the temperature dT is changed to equation (8); for $\mathrm{dR}$ equation (7) can be used.

$$
\mathrm{d} T=\frac{\frac{k_{3}}{k_{2}} \cdot U_{2}+U_{3}}{a \cdot I_{5} \cdot k_{3} \cdot R_{0}}-\frac{1}{a}
$$

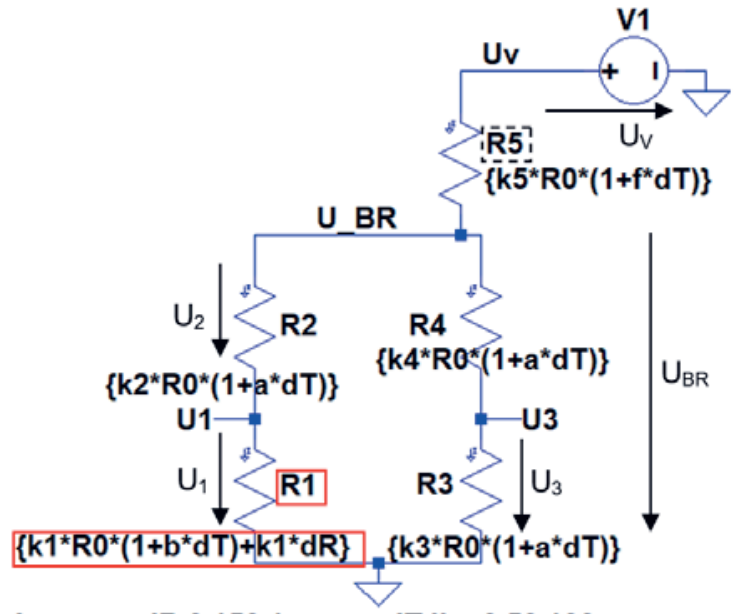

dc param dR 01501 param dT list 050100

Fig. 6: Quarter bridge circuit with additional resistor $R 5$ and a bridge circuit with one sensor element (R1) and three reference resistors $(R 2, R 3, R 4)$

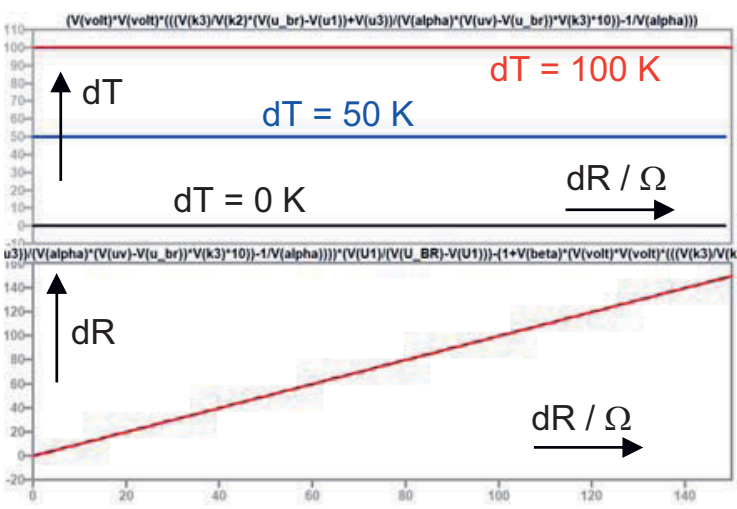

Fig. 7: Quarter bridge circuit (see Fig. 6) depending on change of $R(d R)$ with temperature change $d T$ as parameter: Top: Temperature change $d T$ Bottom: Resistance change $d R$

\subsection{Half bridge circuit with two sensor elements and additional series resistor}

Figure 8 shows a half bridge circuit with two sensor elements (R1 and R4), two reference resistors (R2 and R3) and additionally the resistor $\mathrm{R} 5$ in series connection to the bridge circuit. The temperature coefficient $f$ of the series resistor R5 must differ from the temperature coefficient $a$ of $\mathrm{R} 2$ and $\mathrm{R} 3$ (hence: $f \neq a$ ), but it is possible: $a=b$ with $b$ as temperature coefficient of R1 and R4. 


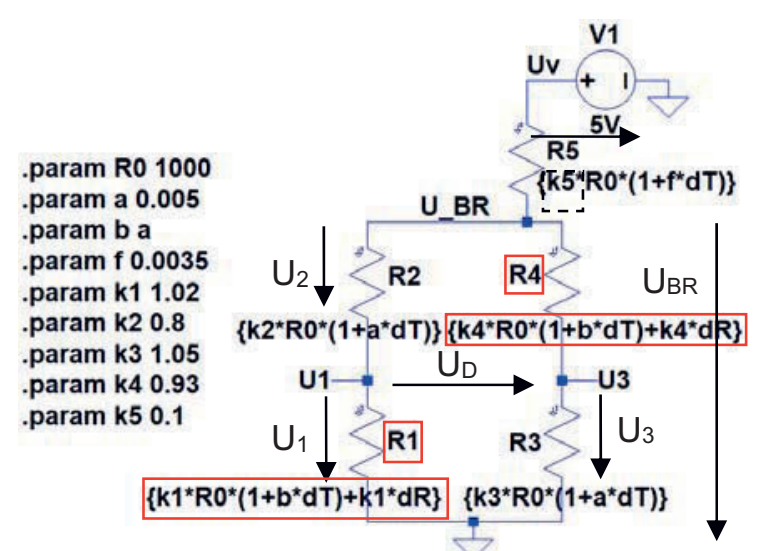

.dc param dR 01501 param dT list 050100

Fig. 8: Half bridge circuit with additional resistor $R 5$ and a bridge circuit with two sensor elements (R1, R4) and two reference resistors $(R 2, R 3)$

Equation (9a) applies to the change in temperature dT under the condition that the value of R5 is constant (possible: $a=b$ ) and equation (9b) applies to dT if R5 (with $f$ as constant temperature coefficient) is at the same temperature as the bridge circuit; dT can be used to calculate $d R$ (or $d R / R_{0}$ ) in equation (10)

$$
d T=\frac{\frac{k_{3}}{k_{2}} \cdot U_{2}+U_{3}}{a \cdot\left(U_{V}-U_{B R}\right) \cdot \frac{k_{3} \cdot R_{0}}{R_{5}}}-\frac{1}{a} \quad \text { (9a): with }
$$

R5 as constant resistor

and equation (9b) with $\mathrm{f}$ as TC of R5:

$$
\begin{aligned}
& d T=\frac{k_{2} \cdot k_{3} \cdot U_{5}-k_{2} \cdot k_{5} \cdot\left(U_{B R}-U_{D}\right)}{\left[k_{2} \cdot k_{5} \cdot\left(U_{B R}-U_{D}\right)+k_{5} \cdot U_{2} \cdot\left(k_{3}-k_{2}\right)\right] \cdot f} \\
& \ldots \frac{-k_{5} \cdot\left(U_{B R}-U_{1}\right) \cdot\left(k_{3}-k_{2}\right)}{-k_{2} \cdot k_{3} \cdot U_{5} \cdot a} \\
& \frac{d R}{R_{0}}=\frac{U_{B R} \cdot k_{3}-U_{3} \cdot\left(k_{3}+k_{4}\right)}{U_{3} \cdot k_{4}}+ \\
& +\frac{d T \cdot\left[U_{B R} \cdot k_{3} \cdot a-U_{3} \cdot\left(a \cdot k_{3}+b \cdot k_{4}\right)\right]}{U_{3} \cdot k_{4}}
\end{aligned}
$$

In Figure 9 the results of equation (9b) and (10) are depicted. The temperature change dT and the change of the sensor resistance $\mathrm{dR}$ can be determined again simultaneously. Similar to the previous chapter, the voltage source V1 and the resistor R5 can be replaced by a current source 15; then equation (8) is valid again.

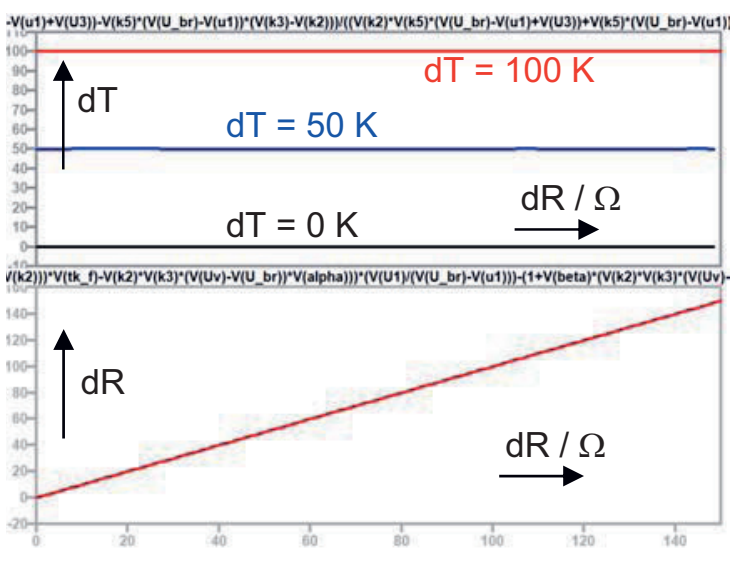

Fig. 9: Half bridge circuit (see Fig. 8) depending on change of $R(d R)$ with temperature change $d T$ as parameter: Top: Temperature change $d T$ Bottom: Resistance change $d R$

\subsection{Full bridge circuit with four sensor elements and additional series resistor}

A full bridge circuit consists of four sensor elements as shown in Figure 10. Then the two sensor elements R1 and R4 must show reciprocal behaviour compared to the other two sensor elements R2 and R3, so that the resistance of the bridge circuit can be assumed to be constant - only depending on temperature change dT. Equation (11a) applies to the change in temperature dT provided that the value of R5 is constant; equation (11b) applies to dT when R5 is [R5 $\left.=k 5^{*} R_{0}{ }^{*}\left(1+f^{*} d T\right)\right]$, where $f$ is the constant temperature coefficient of R5 at the same temperature as the bridge circuit; $f$ must be different to $b$ as TC of the sensor elements $(\rightarrow f \neq b$ ); equation (12) can be used to calculate $d R$ or the relative change $d R / R_{0}$.

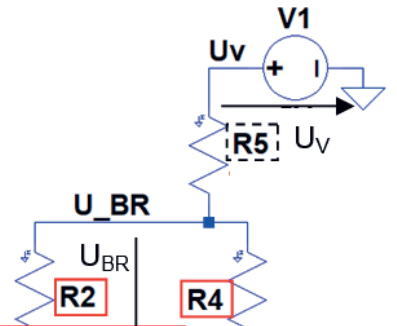

$\left\{k 2{ }^{*} 0^{*}\left(1+b^{*} d T\right)-k 2 * d R\right\}\left\{k 4^{*} R 0^{*}\left(1+b^{*} d T\right)+k 4^{*} d R\right\}$

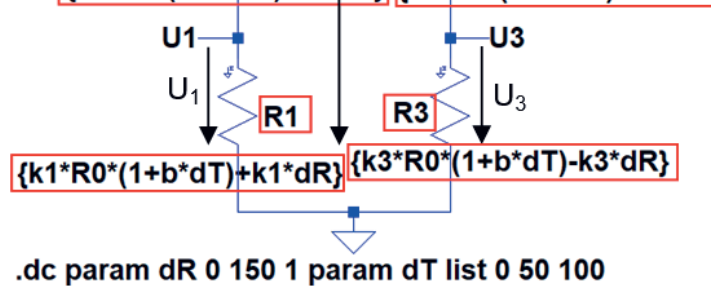

Fig. 10: Full bridge circuit with four sensor elements (R1, R2, R3, R4) and an additional resistor $R 5$ 
Equation (11a) with $\mathrm{R} 5$ as constant results in $d T=\frac{U_{B R} \cdot\left(k_{1}+k_{2}+k_{3}+k_{4}\right) \cdot R_{5}}{b \cdot\left(U_{V}-U_{B R}\right) \cdot R_{0} \cdot\left(k_{1}+k_{2}\right) \cdot\left(k_{3}+k_{4}\right)}-\frac{1}{b}$

and equation (11b) with $\mathrm{f}$ as TC of R5

$$
\begin{aligned}
d T= & \frac{\left(U_{V}-U_{B R}\right) \cdot\left(k_{1}+k_{2}\right) \cdot\left(k_{3}+k_{4}\right)+}{U_{B R} \cdot\left(k_{1}+k_{2}+k_{3}+k_{4}\right) \cdot k_{5} \cdot f+} \\
& \ldots \frac{-U_{B R} \cdot\left(k_{1}+k_{2}+k_{3}+k_{4}\right) \cdot k_{5}}{-b \cdot\left(U_{V}-U_{B R}\right) \cdot\left(k_{1}+k_{2}\right) \cdot\left(k_{3}+k_{4}\right)} \\
\frac{d R}{R_{0}}= & \frac{\left[U_{B R} \cdot k_{1}-U_{1} \cdot\left(k_{1}+k_{2}\right)\right] \cdot(1+b \cdot d T)}{U_{1} \cdot\left(k_{1}-k_{2}\right)-U_{B R} \cdot k_{1}}
\end{aligned}
$$

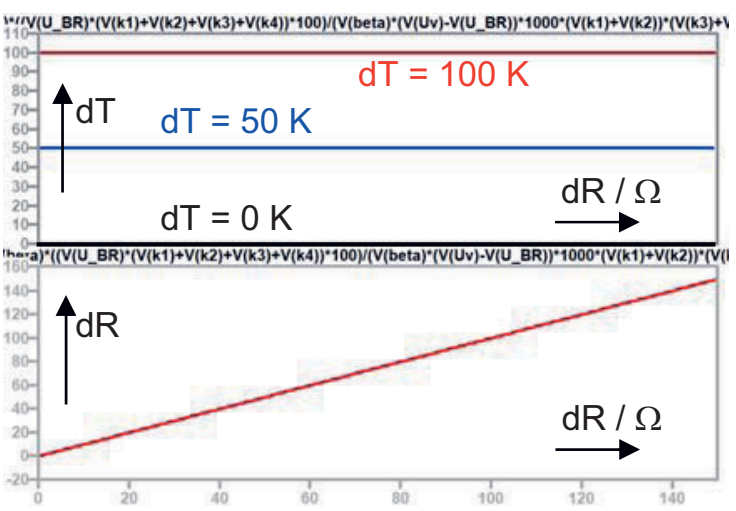

Fig. 11: Full bridge circuit (see Fig. 10) depending on change of $R(d R)$ with temperature change $d T$ as parameter: Top: Temperature change dT Bottom: Resistance change $d R$

Figure 11 shows the results for $\mathrm{dT}$ (equation (11a)) and for dR according to equation (12).

Similar to the previous chapter, the voltage source $\mathrm{V} 1$ and the resistor $\mathrm{R} 5$ can be replaced by a current source $\mathrm{I}_{5}$, then the equation (11c) applies instead of the equation (11a) or (11b). Equation (11c) with current source 15 instead of voltage source $\mathrm{V} 1$ results in:

$$
d T=\frac{U_{B R} \cdot\left(k_{1}+k_{2}+k_{3}+k_{4}\right)}{b \cdot I_{5} \cdot R_{0} \cdot\left(k_{1}+k_{2}\right) \cdot\left(k_{3}+k_{4}\right)}-\frac{1}{b}
$$

\section{Method for simultaneously determining the change in resistances and temperature in a Wheatstone bridge using AMR sensor elements}

This example shows the new concept with an AMR sensor element implemented in LTspice [3] as variable resistor. The model for the AMR sensor element is described in [2]. Here the temperature coefficient (TC) of the AMR sensor element is $+0.003 / \mathrm{K}$ (relevant for $\mathrm{R}_{0}$ ) and the TC of the AMR effect is $-0.002 / \mathrm{K}$ (relevant for $\mathrm{dR}$ ).

\subsection{Quarter bridge circuit using one AMR sensor element}

In Figure 12 a quarter bridge circuit with one AMR sensor element R1 is shown. The TC of $\mathrm{R} 3$ and $\mathrm{R} 4$ must be different $(\mathrm{a} \neq \mathrm{g})$; $\mathrm{a}$ as TC of R3 may be equal to $b$ as TC of the AMR sensor element $(a=b)$, e.g. by using an insensitive AMR-sensor for R3. The change in temperature dT can be derived using equation (13) which is similar to equation (2). As depicted in Figure 13, the typical AMR characteristic ( $R$ depending on the magnetic field strength $H$ in the direction of the hard axis) is influenced by the temperature change dT: The higher dT, the lower the change of the resistance $\mathrm{dR}$ due to the negative TC $(-0.002 / \mathrm{K})$ of the AMR effect.

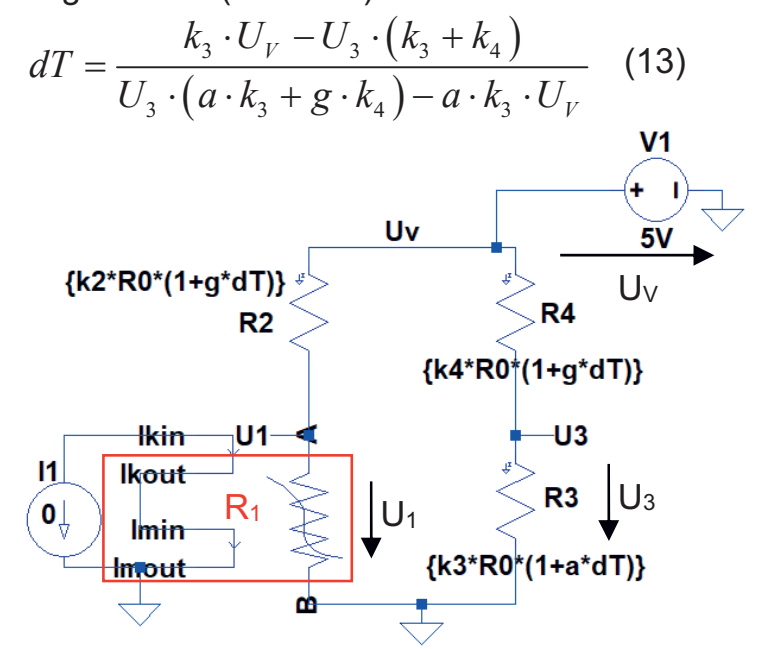

Fig. 12: Quarter bridge circuit with one sensor element (AMR-sensor R1) and three reference resistors

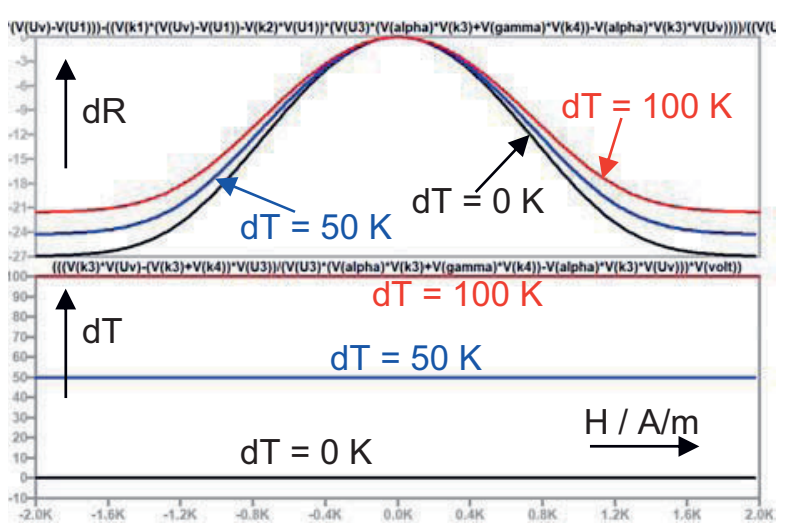

Fig. 13: Quarter bridge circuit (see Fig. 12) depending on applied magnetic field strength $H$ with temperature change dT as parameter. Top: Resistance change dR; Bottom: Temperature change $d T$ 


\subsection{Full bridge circuit using four AMR sensor elements}

In Figure 14 a full bridge circuit with four AMRsensor elements R1 to R4 is depicted. The resistors R1 and R4 must exhibit opposite behaviour to R2 and R3, which can be achieved by barber pole structures. In Figure 15 the results of the simulation is depicted: The temperature change dT (see equation (14)), the resistance change $\mathrm{dR}$ and also the output voltage $U_{D}$ can be determined simultaneously using the corresponding equations.

Equation (14) is valid for the full bridge circuit under the condition that R5 is constant (see equation (11a)):

$d T=\frac{U_{B R} \cdot\left(k_{1}+k_{2}+k_{3}+k_{4}\right) \cdot R_{5}}{b \cdot\left(U_{V}-U_{B R}\right) \cdot R_{0} \cdot\left(k_{1}+k_{2}\right) \cdot\left(k_{3}+k_{4}\right)}-\frac{1}{b}$

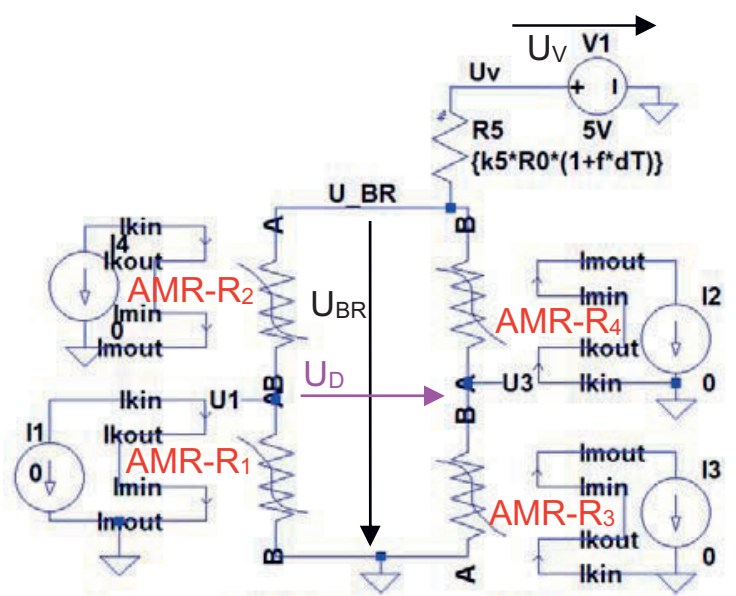

.dc param H -2000200025 param dT list 050100

Fig. 14: Full bridge circuit with four sensor elements (AMR-sensors R1-R4)

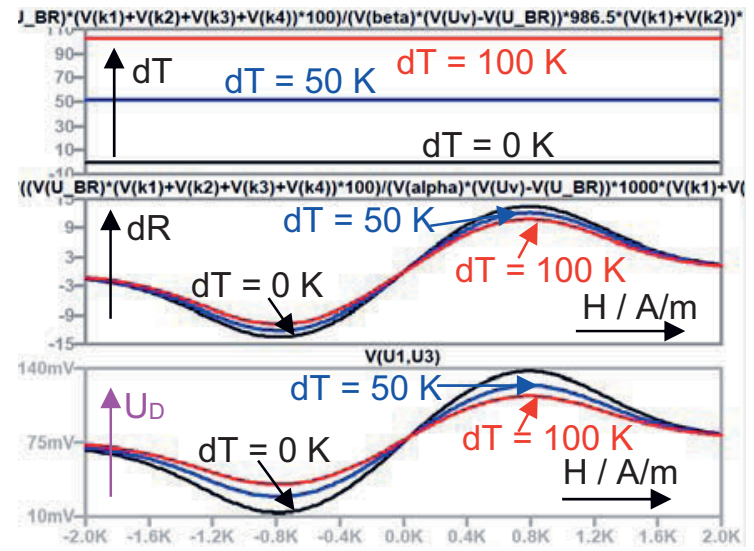

Fig. 15: Full bridge circuit (see Fig. 14) depending on applied magnetic field strength $H$ with temperature change $d T$ as parameter. Top: Resistance change dR; middle: Temperature change $d T$; bottom: Voltage $U_{D}$

\section{Conclusion}

A new concept is described which allows the simultaneous measurement of the change in resistance $d R$ and the change in temperature $\mathrm{dT}$. This is achieved by using the applied voltages and temperature coefficients of all resistors in the Wheatstone bridge. This concept is applicable to quarter, half and full bridge circuits with different numbers of sensor elements. It is shown how the relevant information (resistors R1 to R4) is determined in an already manufactured Wheatstone bridge circuit with an operational amplifier circuit. A possible offset of the bridge circuit due to a mismatch of the four resistors is not relevant. The additional information about the temperature can help to obtain more accurate results. The bridge circuit can not only be used for compensation of the temperature influence, but also as a temperature sensor for condition monitoring or diagnostic functions in the application. Thus, the bridge circuit can provide two different information simultaneously: resistance change $\mathrm{dR}$ and the temperature change $\mathrm{dT}$. In this example, the temperature coefficient is assumed to be constant - if this is not the case, the calculation can be performed using a numerical approach. A patent application has already been filed.

\section{References}

[1] Tietze, U.; Schenk, Ch.: Halbleiterschaltungstechnik, 9. Auflage, Springer-Verlag, Berlin Heidelberg, 1990, S. $864 f$

[2] Gintner, K.: Ein Sensor auf Basis des anisotropen magnetoresistiven Effekts, Dissertation, Lehrstuhl für Technische Elektronik, Friedrich-Alexander-Universität Erlangen-Nürnberg, 1999

[3] Engelhardt, M.: LTspice XVII(x64), 2018, Linear Technology Corporation 